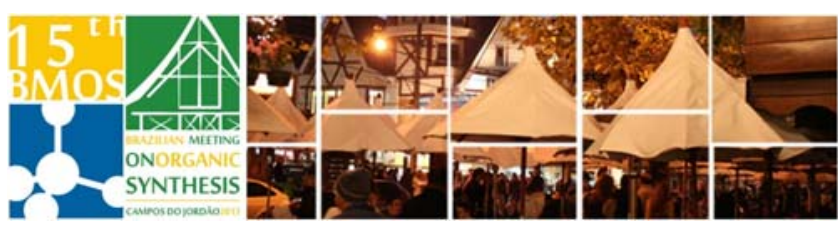

\title{
Synthesis and Functions of Biomimetic Helical Polymers and Oligomers
}

\author{
Eiji Yashima \\ Department of Molecular Design and Engineering, Graduate School of Engineering, \\ Nagoya University, Chikusa-ku, Nagoya 464-8603, Japan \\ *e-mail yashima@apchem.nagoya-u.ac.jp
}

Keywords: Helix, Chiral recognition, Supramolecules

\section{Abstract Speech}

Unique macromolecules and oligomers that fold into a preferred-handed single- or double-stranded helical conformation induced by chiral substituents covalently bonded to the main-chains or external chiral stimuli followed by memory of the helical chirality are presented. ${ }^{1}$ The direct observations of helical structures of artificial helical polymers by atomic force microscopy (AFM) will be also presented. ${ }^{2}$ A series of double helices composed of different components and sequences that exhibit specific functions, such as chirality sensing, chiral recognition, enantioselective asymmetric catalysis, and anisotropic spring-like motion are also described. $^{3}$

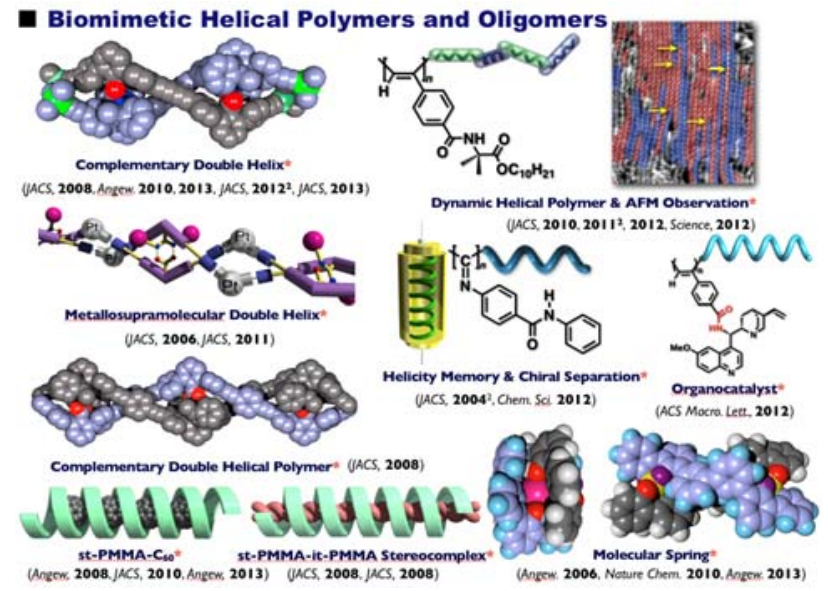

Figure Single- and double-stranded helical polymers and oligomers with specific functions.

\section{REFERENCES}

${ }^{1}$ Yashima, E.; Maeda, K.; lida, H.; Furusho, Y.; Nagai, K. Chem. Rev. 2009, 109, 6102.

${ }^{2}$ Kumaki, J.; Sakurai, S.-i.; Yashima, E. Chem. Soc. Rev. 2009, 38, 737.

${ }^{3}$ Furusho, Y.; Yashima, E. Macromol. Rapid Commun. 2011, 32, 136 